Sergio Ortolani did his thesis on the dynamics of fast z-pinches at the ENEA Fusion Laboratory in Frascati (Rome), receiving his doctorate in Physics from the University of Rome in 1970. In 1971, he joined the Istituto Gas Ionizzati of the Italian National Council of Research (CNR) in Padova where he began studying plasma magnetic confinement in Reversed Field Pinch configurations as part of the European Fusion Programme. In 1975 he worked at the CTR division of Los Alamos National Laboratory on toroidal pinch experiments. For the last 20 years he has been engaged in experimental and theoretical research on MHD fluctuations and on plasma radiation and transport phenomena, working in close collaboration with many research institutions in Europe, USA, Japan, and USSR. He is currently involved in the study of relaxation phenomena and of heating and transport processes in laboratory and in naturally occurring plasmas. Dr. Ortolani is presently CNR Director of Research at the Istituto Gas Ionizzati (EURATOM-ENEA-CNR Association, Padova) where he heads the scientific programme of the newly-constructed European experiment, RFX.

Dalton D. Schnack received his Ph. D. in Applied Science from the University of California, Davis, in 1977. He did his doctoral research at Lawrence Livermore National Laboratory (LLNL), where he began his interest in non-linear MHD processes in fusion plasmas. From 1973 to 1980 he served as a staff physicist in the computational physics group at the National Magnetic Fusion Energy Computer Center at LLNL. In 1980 he joined the fusion theory group at Los Alamos National Laboratory, where he worked on problems relevant to the reversed field pinch and compact torus experiments. In 1982 he joined Science Applications International Corporation, and in 1986 he was appointed Manager of the Applied Plasma Physics and Technology Division. Dr. Schnack has authored many papers in the fields of linear and non-linear resistive MHD, and computational methods related to such problems. He is actively involved in studying the non-linear MHD properties of the reversed field pinch and the solar corona, in theoretical studies of radiative instabilities in magnetized plasma, and in studying the highly non-linear (turbulent) properties of the Navier-Stokes and MHD equations. 
This page is intentionally left blank

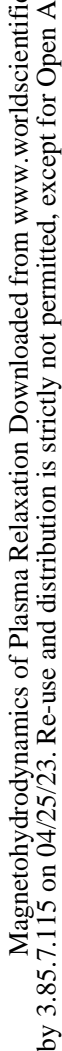




\title{
Magnetohydrodynamics of Plasma Relaxation
}

\section{Sergio Ortolani}

\author{
Istituto Gas Ionizzati CNR
}

EURATOM-ENEA-CNR Association

Padova, Italy

\section{Dalton D. Schnack}

Science Applications International Corporation

San Diego, California, USA 


\section{Published by}

World Scientific Publishing Co. Pte. Ltd.

P O Box 128, Farrer Road, Singapore 9128

USA office: Suite 1B, 1060 Main Street, River Edge, NJ 07661

UK office: 73 Lynton Mead, Totteridge, London N20 8DH

\section{MAGNETOHYDRODYNAMICS OF PLASMA RELAXATION \\ Copyright @1993 by World Scientific Publishing Co. Pte. Ltd. \\ All rights reserved. This book, or parts thereof, may not be reproduced in any form or by any means, electronic or mechanical, including photocopying, recording or any information storage and retrieval system now known or to be invented, without written permission from the Publisher.}

ISBN $981-02-0860-\mathrm{X}$

Printed in Singapore by JBW Printers \& Binders Pte. Ltd. 


\section{Contents}

PREFACE

1. INTRODUCTION

1.1 Taylor's Theory of Plasma Relaxation ............................................ 2

1.2 The Underlying Physics of Plasma Relaxation................................... 6

1.3 Toroidal Systems and the RFP............................................................ 9

1.4 Overview of the Remainder of the Book ........................................ 12

2. THE RESISTIVE MAGNETOHYDRODYNAMIC MODEL...................... 15

2.1 Resistive Magnetohydrodynamics................................................... 15

The Physical Model ........................................................................ 15

The Evolution of the Fluid.............................................................. 17

The Evolution of the Electromagnetic Fields................................ 21

Characteristic Oscillations; Normal Modes..................................... 22

Dimensionless Variables; the Lundquist Number.......................... 25

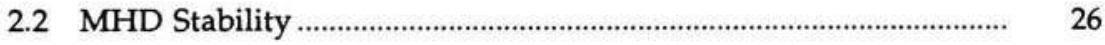

Linear Stability of Normal Modes................................................ 26

Magnetic Shear and Singular Surfaces......................................... 28

Toroidal Pinch Configurations..................................................... 29

Resistive Instabilities ................................................................... 31

Nonlinear Effects ......................................................................... 37

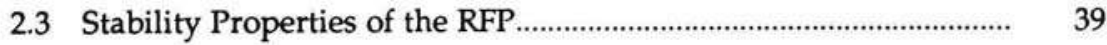

2.4 The Force Free MHD Model...…………………………………..... 42

2.5 The Role of Numerical Simulation ................................................. 43

3. TAYLOR'S THEORY OF PLASMA RELAXATION ……......................... 47

3.1 The Constraints of Ideal MHD....................................................... 48

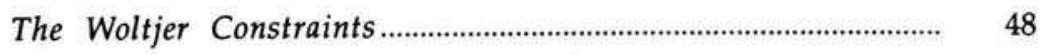

The Topological Properties of the Woltjer Constraints................ 50

3.2 Energy Minimization with the Constraints of Ideal MHD............. 51 
3.3 The Effect of Plasma Resistivity....................................................... 53

Taylor's Conjecture .......................................................................... 53

3.4 Energy Minimization with the Global Helicity Constraint........... 54

Validity of Taylor's Conjecture......................................................... 55

Properly Defined Helicity............................................................... 57

The Role of Plasma Pressure in Taylor's Theory............................ 58

3.5 Predictions of the Theory.................................................................. 58

The Reversed-Field Pinch................................................................. 58

Summary of RFP Predictions.......................................................... 61

The Multipinch Experiment ......................................................... 62

3.6 Discussion........................................................................................... 63

4. PHENOMENOLOGY OF RELAXATION IN THE REVERSED-FIELD

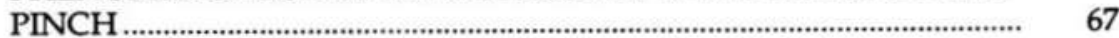

4.1 Mean Field Profiles.................................................................................. 68

4.2 The Stability of Relaxed States ……..................................................... 74

4.3 Resistive Diffusion ................................................................................... 82

4.4 The Phenomenological Cyclical Model ......................................... 87

4.5 Experimental Observations of Relaxation Phenomena in the RFP......................................................................................... 88

5. THE DYNAMICS OF PLASMA RELAXATION...................................... 95

5.1 Classical Dynamo Theory.................................................................. 96

Kinematic Dynamos......................................................................... 97

Cowling's Theorem............................................................................ 99

The Turbulent Dynamo .................................................................. 101

Relevance to the RFP Dynamo.................................................... 102

5.2 The Basic Relaxation Mechanism .................................................. 104

The Original Work of Sykes and Wesson...................................... 104

Spontaneous and Driven Reconnection in the RFP..................... 106

Fluctuations and Ohm's Law........................................................ 110

Evidence of Taylor Relaxation ....................................................... 111 
The Helical Ohmic State.

5.3 Effects of Nonlinear Mode Coupling.

MHD Fluctuations.

Nonlinear Mode Coupling.

5.4 Summary.

6. PRACTICAL ISSUES RELATED TO RELAXATION

6.1 Anomalous Loop Voltage.

Perfectly Conducting Outer Boundary

Operation with Resistive Walls and Limiters.

Helicity Balance.

6.2 Taming the Dynamo; An Application of the Theory

7. RELAXATION AND THERMAL TRANSPORT.

7.1 A Model for Sawtooth Oscillations in the RFP.

Experimental Observations.

Theoretical Interpretation of the Sawtooth Crash.

7.2 Thermal Transport During Sawtooth Oscillations

Energy Confinement Time.

Modifications to the Resistive MHD Model

Simulation of Sawtooth Oscillations

7.3 Summary.

8. DYNAMICAL RELAXATION IN THE SOLAR CORONA..................... 155

8.1 Overview of Coronal Dynamics...................................................... 155

8.2 Magnetic Arcade Evolution.............................................................. 157

8.3 Coronal Current Filaments ................................................................ 163

8.4 An Analogy Between the Solar Corona and the RFP.................. 170

9. SUMMARY …............................................................................................. 171

9.1 Relaxation in the Reversed-field Pinch......................................... 172

9.2 Relaxation and Transport ................................................................. 174

9.3 Relaxation in the Solar Corona ..................................................... 175 
viii Contents

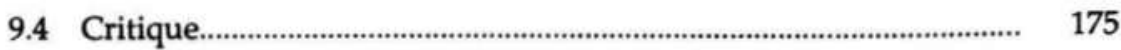

To what extent can the numerical simulations be believed?....... 176

What about analytic theory? ............................................................. 176

What is the role of turbulence? ....................................................... 176

Are pressure driven modes important? .......................................... 177

Are there non-MHD effects? ........................................................... 177

What is the future of relaxation studies? ....................................... 177

REFERENCES .................................................................................................. 179

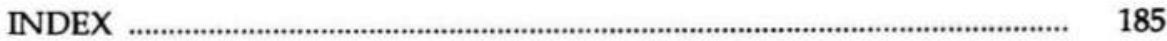




\section{Preface}

This book describes what the authors have learned about the causes and consequences of plasma relaxation over the past twenty years. Much of this knowledge has come from the field of controlled fusion research, in particular the Reversed-field Pinch (RFP) concept. Thus, a certain bias toward plasma phenomena inherent in this experiment is inevitable. However, it is the authors' belief that these phenomena are sufficiently generic that the concepts presented herein will be useful to researchers in other fields. Failing that, the book is at least an account of a successful world-wide research program that has led to a fairly complete, self-consistent description of a particular type of hot, magnetized plasma.

Early laboratory experiments discovered that axisymmetric toroidal pinch plasma discharges exhibited favorable confinement properties when operated in one of two regimes: the tokamak, in which the toroidal component of the magnetic field was approximately uniform and much larger than the poloidal component; and, the RFP, in which the toroidal field reversed direction in the outer regions of the plasma, and was comparable to the poloidal field. Furthermore, it was found that these states could be formed spontaneously by controlling only a few global parameters, such as the total toroidal current and magnetic flux. This behavior was explained in a general theoretical way by J. B. Taylor in 1974. Taylor used a variational principle with constraints to show that these states were the preferred states of the magnetoplasma system. These states were called relaxed states, and the process of attaining them was called relaxation. Relaxation concepts have since been applied in a variety of situations by a number of researchers to explain the natural states of plasmas and fluids.

The variational theory has proven useful for predicting the time-asymptotic average steady state of many systems, but it does not directly address the fundamental physical processes that give rise to plasma relaxation. Thus, in the ensuing years the fundamental questions shifted from explaining the existence of preferred states to understanding how and why relaxation occurs. Answering these questions has required a synergism between detailed experimental measurements, large scale numerical computations, and theoretical plasma physics. The results of these efforts are the subject of this book.

Taylor's theory requires only that the dynamical processes responsible for plasma relaxation be describable by the equations of resistive magnetohydrodynamics (MHD). While the specific details of the dynamics do not enter the variational theory, Taylor envisioned plasma relaxation as 
the result of MHD turbulence. These fluctuations are characterized by relatively high frequency and short spatial scales. Subsequent experiments showed that the ubiquitous characteristic fluctuations in RFP discharges have low frequency and broad spatial extent. These fluctuations were reproduced in large scale computer simulations of RFP discharges. These simulations also exhibited plasma relaxation. Theoretical analyses of these results have shown how these long wavelength MHD fluctuations arise, and how they can produce not only the preferred relaxed states predicted by the variational theory, but can also account for many of the operating characteristics of these experiments. Further calculations have suggested that the dynamics that underlie plasma relaxation may also play an important role in determining the energy confinement properties of RFP plasmas.

The presentation of these results is (we hope) logical rather than historical, and relatively self-contained. Chapter 1 is devoted to a brief overview of plasma relaxation, toroidal pinches in general, and the RFP in particular. Chapter 2 presents the fundamentals of the resistive MHD model as required for describing plasma relaxation, including discussions of ideal and resistive instabilities and nonlinear phenomena. Taylor's variational theory is presented in detail in Chapter 3. In Chapter 4 we present phenomenological models of plasma relaxation as deduced from experimental observations and linear stability theory. Typical experimental results are also presented. The results of detailed, large scale numerical simulations of the magnetic field dynamics in RFP plasmas are presented in Chapter 5. It is these calculations that self-consistently explain both the presence of the experimentally observed long-wavelength fluctuations and their role in producing plasma relaxation. A brief discussion of the so-called RFP dynamo is also given here. The remainder of the book is devoted to the consequences of plasma relaxation, and to relaxation in situations other than fusion experiments. In Chapter 6 we show that the same fluctuations that are responsible for the attainment of relaxed states can also self-consistently account for anomalous plasma resistance, and enhanced loop voltage when discharges are operated with resistive shells and limiters. Chapter 7 contains a discussion of the possible consequences that the inherent fluctuations may have for energy transport and confinement. Chapter 8 describes calculations of similar dynamical events that may occur in the solar corona, the magnetized outer atmosphere of the sun. While the boundary conditions make it difficult to make a direct connection between these phenomena and the variational relaxation theory, similarities between the dynamics of the coronal and RFP plasmas are noted and discussed. A brief summary is found in Chapter 9.

Many researchers have contributed to the picture of plasma relaxation that is presented in this book. To attempt to acknowledge them all would be folly, for some deserving soul would surely be left off any list. Nonetheless, 
the authors would like to especially thank their longtime colleagues H. A. B. Bodin, E. J. Caramana, J. Killeen, and R. A. Nebel for providing both insights and a stimulating scientific environment. We wish to acknowledge the specific contributions of our close collaborators V. Antoni, D. C. Barnes, S. Cappello, G. G. Craddock, D. S. Harned, Y. L. Ho, P. Martin, D. Merlin, Z. Mikik, and R. Paccagnella. We would also like to thank S. Prager and C. Gimblett for carefully reading a draft version of the manuscript and providing valuable comments that greatly improved the clarity and accuracy of the presentation. We thank CNR of Italy and the United States Department of Energy for supporting this research over the years. We are indebted to Ms. Anna Miklovic for the seemingly endless task of formatting and preparing the camera-ready manuscript. This book exist because of her persistent and professional efforts. One of us (DS) thanks Prof. C. K. Rowdyshrub for spiritual enlightenment. Finally, we emphasize that the interpretation and synthesis of the results presented here is strictly the viewpoint of the authors.

\author{
Sergio Ortolani \\ Padova, Italy \\ Dalton D. Schnack \\ San Diego, CA
}

July 1992 